\title{
Simulation Study on Internal Transparent Collector IGBT Using Superjunction Drift
}

\author{
Dongqing $\mathrm{Hu}$, Peihao Lv, Yunpeng Jia \\ College of Electronic Information and Control Engineering \\ Beijing University of Technology \\ Beijing, China \\ e-mail: hudongqing@bjut.edu.cn
}

\begin{abstract}
The super junction (SJ) drift is used in internal transparent collector IGBT. The influences of position of local carrier lifetime region, local carrier lifetime, and buffer layer doping level on the devices' trade-off characteristics have been simulation studied. The results show that for given local carrier liftetime, the turn-off losses may have a valley value $E_{\text {offmin }}$. The higher local carrier lifetime is, the higher $E_{\text {offmin }}$ will be. Several factors are at work. Competition effects for excess carrier extraction through reverse biased super junction and forward biased collector may be one reason. Infinite excess carrier recombination velocity in local carrier lifetime control region leads to the back-emitter injection efficiency $\gamma_{e}$ be no less than certain value. In addition; increasing buffer layer doping level appropriately could win better trade-off and wider trade-off range.
\end{abstract}

Keywords- superjunction; local carrier lifetime; internal transparent collector; IGBT

\section{INTRODUCTION}

MOSFET is widely used in low and mid voltage application. But as a unipolar device, the specific drift region on-state resistance $\mathrm{s} R_{\text {don }}$ has 2.5-power dependence on breakdown voltage $\mathrm{e}^{[1]}: s R_{\text {on }} \propto(B V)^{2.5}$. So the on-state resistance becomes so large for high voltage MOSFET. This limits its application in high voltage fields. To overcome this limitation, superjunction (SJ) drift region based on charge balance is proposed ${ }^{[2]}$ and first used in CoolMOS ${ }^{[3]}$. SJ drift region makes the drift region resistance is nearly linear dependent on blocking voltage $\mathrm{e}^{[4-5]}$. This makes the resistance of high voltage MOSFET reduced greatly.

Around 2002, SJ concept is used in planar PT-IGBT ${ }^{[6]}$. Simulation results show SJ FS-IGBT has the best tradeoff performance in its class. But for 600V IGBT, FS-IGBTs need super thin wafer technology. So most studies on low voltage SJ FS-IGBT are focused on simulation research ${ }^{[7-10]}$.

Internal transparent collector (ITC) IGBT is a new type of IGBT proposed for low voltage IGBT (for example $600 \mathrm{~V})^{[11]}$. It based on PT-IGBT technology, but a local carrier lifetime control (LCLC) region is introduced in the collector region near the buffer-collector junction. This changes the collector from non-transparent to internal transparent. The device has similar performance to that of transparent collector IGBT, but needs no thin wafer technology for low voltage IGBT. In this paper, SJ drift region is introduced in ITC-IGBT, and SJ ITC-IGBT is obtained. Though SJ ITC-IGBT may have some similar behaviors to SJ FS-IGBT, finite recombination rate of local carrier lifetime region makes its performance be influenced by local carrier life and the position of LCLC region. Thus, SJ ITC-IGBT has its own characteristic and particularity. In this paper, trade-off performance of $600 \mathrm{~V}$ SJ ITC-IGBT under different local carrier lifetime and LCLC position will be simulation studied. In addition, the influence of buffer layer doping level are also studied.

\section{STRUCTURE OF SJ ITC-IGBT}

Simulation used structure of $600 \mathrm{~V}$ planar gate SJ ITCIGBT is shown in figure 1 ( half cell).

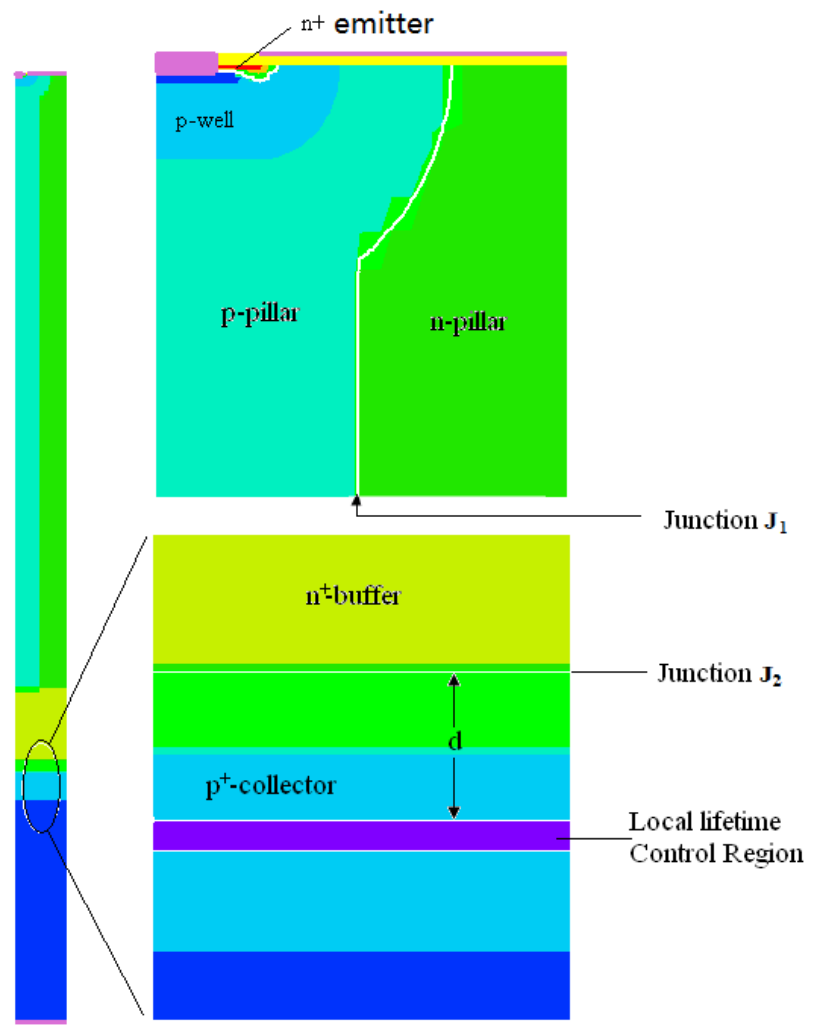

Figure 1 Cross section of SJ ITC- IGBT

The channel is $1.5 \mu \mathrm{m}$ long, $\mathrm{SJ}$ drift region is $50 \mu \mathrm{m}$ long, buffer layer is $5 \mu \mathrm{m}$ thick, and $\mathrm{p}^{+}$substrate doping level is 5 
$\times 10^{19} \mathrm{~cm}^{-3}$. Local carrier lifetime control region is $0.3 \mu \mathrm{m}$ wide. The width of $\mathrm{p}$ - pillar and n-pillar are equal. The pillar doping level is determined by (1):

$$
N \cdot w \approx \frac{\varepsilon_{s} E_{m}}{q}=\frac{\varepsilon_{s} \cdot\left(4010 N^{1 / 8}\right)}{q}
$$

Where $N$ is pillar doping level, $w$ is pillar width, $\varepsilon_{\mathrm{s}}$ is silicon permittivity, and $E_{m}$ is maximum electric field of silicon. For 3 um pillar width, the pillar doping level is about $8 \times 10^{15} \mathrm{~cm}^{-3}$, and $6 \times 10^{15} \mathrm{~cm}^{-3}$ doping level is for 4um pillar width.

\section{DEVICE SIMULATIONS AND DISCUSSION}

Simulations were carried out by using TWB ${ }^{[11]}$. During dynamic simulation, area factor $0.1 \mathrm{~cm}^{2}$ was multiplied. The DC link voltage is $300 \mathrm{~V}$. Turn-off losses were estimated under inductive load at $27^{\circ} \mathrm{C}$. The turn-off current is $20 \mathrm{~A}$.

ITC-IGBT is characterized by local carrier lifetime control. The position of LCLC region and local carrier lifetime play important role in determining device performance. Simulations focused on their influence on device trade-off performance.

\section{A. Influence of Position of Local Carrier Lifetime Control Region}

First, the 4um-wide with $6 \times 10^{15} \mathrm{~cm}^{-3}$ doping level SJ pillar, and $1 \mathrm{~ns}$ of local carrier lifetime are used for the device simulation. The dependence of key parameters(including blocking voltage $B V$, on-state voltage $V_{o n}$, turn-off losses $E_{\text {off }}$, and short circuit current $I_{S C}$ ) on the position of LCLC region(labeled as distance $d$ between LCLC region and buffer-collector junction $\mathrm{J}_{2}$, also see figure 1 ) is depicted in figure 2 . It shows that the device's $B V$ and $V_{o n}$ decrease, and yet the $E_{\text {off }}$ and $I_{S C}$ increase as the distance $d$ between LCLC region and buffer-collector junction is increased, owing to the back-side emitter (p-emitter, collector) injection efficiency $\gamma_{\mathrm{e}}$ (defined as the ratio of hole current to total current at buffer-collector junction $\mathrm{J}_{2}$ ) increases with $d$. These follow well-known rule of the device.

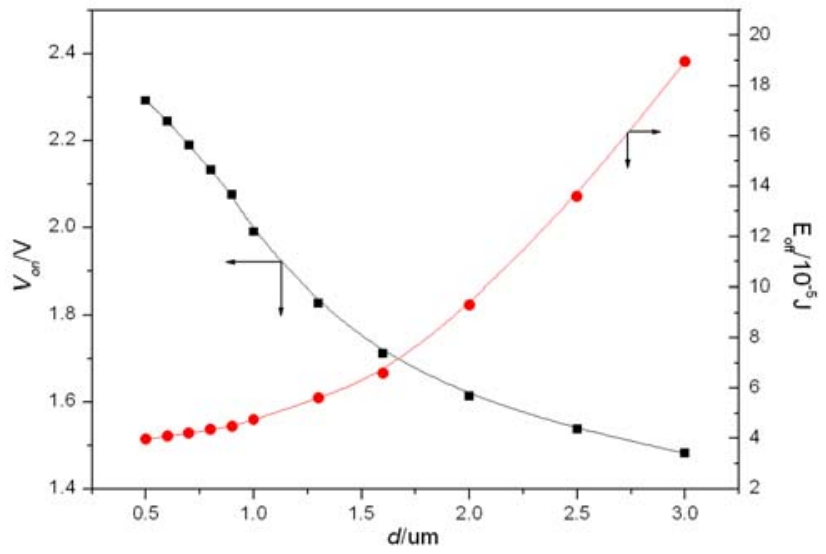

(a) $V_{o n}$ land $E_{o f f}$ dependence on $d$

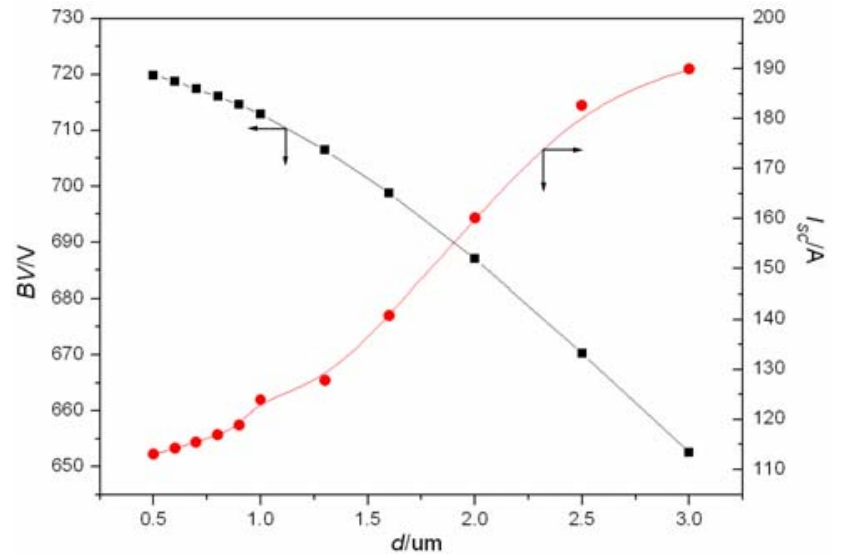

(b) $B V$ and $I_{S C}$ dependence on $d$

Figure 2 Dependence of key parameters on $d$

\section{B. Influence of Local Carrier Lifetime}

To clarify the influence of local carrier lifetime, device trade-off performances under different local carrier lifetime were simulated and the results are shown in figure 3.

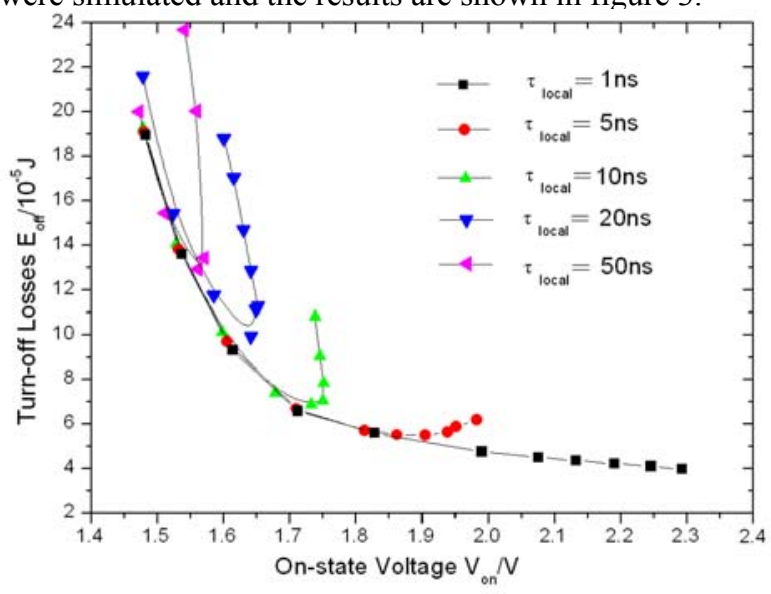

Figure 3 Trade-off curves between $V_{\text {on }}$ and $E_{\text {off }}$ under different local carrier lifetime

The figure shows that: (1) When the local carrier lifetime is short enough, the trade-off curve follows the normal rule that the on-state voltage Von increased and turn-off losses $E_{\text {off }}$ decreased when the distance $d$ between LCLC region and buffer-collector junction is decreased; (2) When the local carrier lifetime increased to some value (for example $5 \mathrm{~ns}$ ), the on-state voltage $V_{\text {on }}$ increased with $d$ decreased, but the turn-off losses decrease first and then increase with $d$ decreased. $E_{\text {off }}$ has a minimum value; (3) Increasing local carrier lifetime further $(>10 \mathrm{~ns})$, both on-state voltage and a turn-off loss has an extreme value when the $d$ varies. The maximum on-state voltage $V_{\text {onmax }}$ and minimum turn-off loss $E_{\text {offmin }}$ changed with the local carrier lifetime. So does the corresponding distance $d_{\text {min }}$ and back-side emitter injection efficiency $\gamma_{e m i n}$. Their relationship is shown in figure 4.

A valley value of $E_{\text {off }}$ also appeared in SJ FS-IGBT ${ }^{[12]}$. It was explained that this phenomenon was due to SJ IGBT 
operation mode change (from bipolar to unipolar). But backemitter injection efficiency controlled by local carrier lifetime is unique to SJ ITC-IGBT. To clarify the mechanism, the back-emitter injection efficiency $\gamma_{e}$ variation with $d$ is plotted in figure 5 .

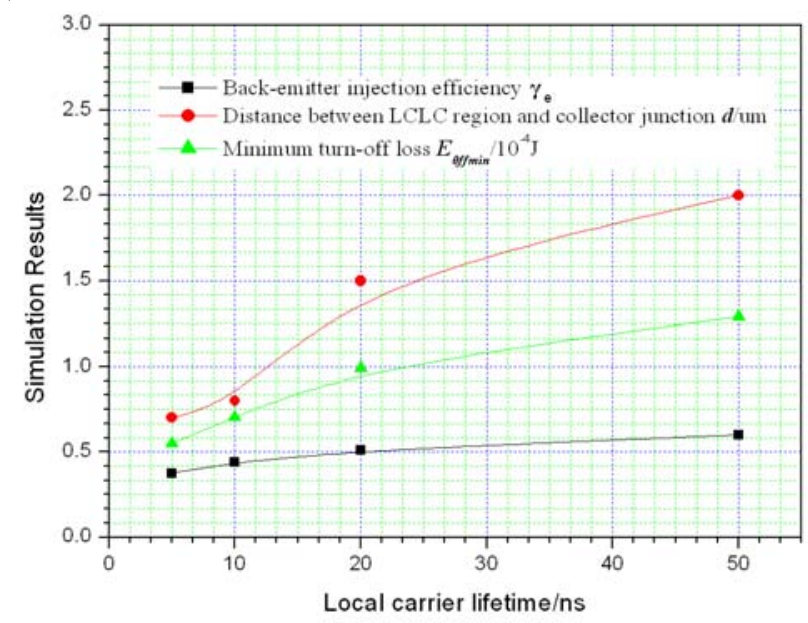

Figure 4 Variation of $E_{\text {offmin }}, d_{\min }$, and $\gamma_{\text {emin }}$ with local carrier lifetime

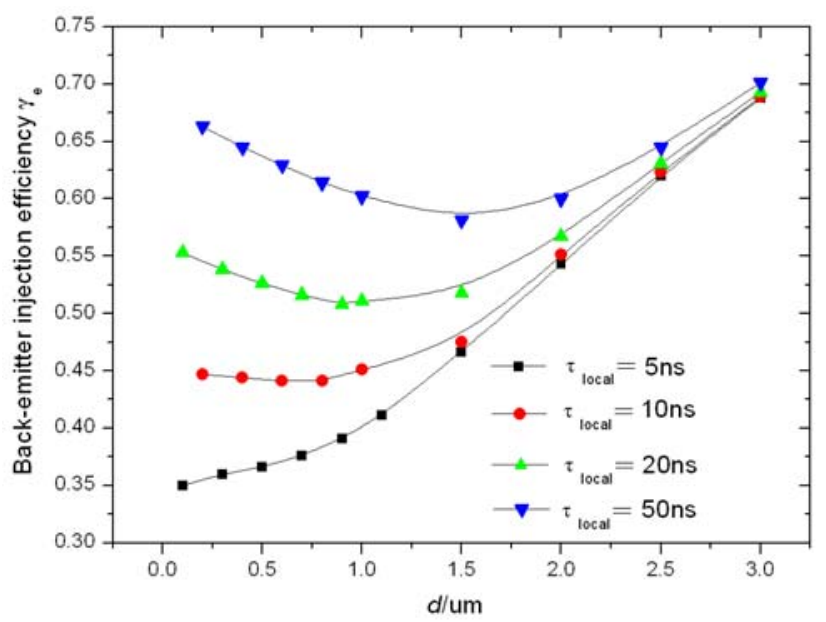

Figure 5 Back-emitter injection efficiency $\gamma_{e}$ changes with $d$.

Figure 5 shows that:

(1) When local carrier lifetime is short (less than $5 \mathrm{~ns}$ ) enough, device back-emitter injection efficiency $\gamma_{e}$ will decreases with $d$. But only the device with 5 ns local carrier lifetime has a turning point at $\gamma_{e}=0.38$. The device with $1 \mathrm{~ns}$ local carrier lifetime has no such point even though the back-side emitter injection efficiency is as low as 0.15 (not plotted in figure 5). So operation mode change may not the only reason which leads to valley turn-off losses. The competition effects for excess carrier extraction through two junctions_reverse biased super junction and forward biased buffer-collector junction, may also influence the turn-off loss. This will be discussed in other paper.

(2) When local carrier lifetime is longer, owing to the finite excess carrier recombination velocity in local carrier lifetime control region, the back-emitter injection efficiency $\gamma_{e}$ has a minimum value. This leads to the minimum $E_{\text {off }}$ and maximum $V_{o n}$. To obtain faster switching speed, the modification of LCLC region position is not enough. Other method such as buffer layer doping level control should be taken. Next section will discuss such question.

In summary, for given local carrier lifetime, the position of LCLC region has an optimum range. Too near of LCLC region to buffer-collector junction may leads to either higher turn-off loss (for longer local carrier lifetime) or higher onstate voltage (for shorter local carrier lifetime).

\section{Influence of Buffer Layer Doping Level}

The buffer layer of IGBT normally has two roles, one is for field stop and the other is for injection efficiency (backemitter) control. Keeping buffer layer thickness unchanged and increasing buffer layer doping level from $5 \times 10^{16} \mathrm{~cm}^{-3}$ to $7 \times 10^{16} \mathrm{~cm}^{-3}$, simulation study the trade-off performance of SJ ITC-IGBT with 10ns local carrier lifetime. The results show that trade-off performance has a little improvement and lower minimum turn-off loss could be obtained comparing to lighter buffer doping level (see figure 6). Increasing buffer layer doping level appropriately could win better trade-off and wider trade-off range.

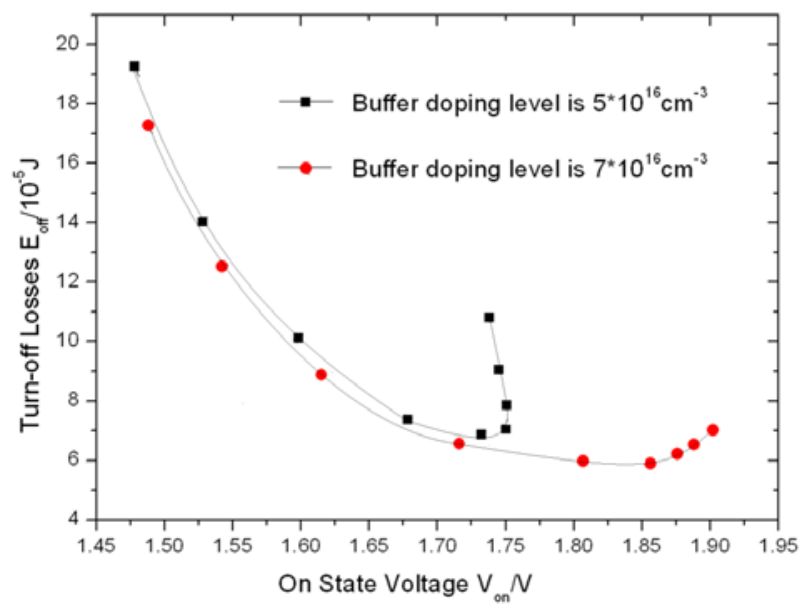

Figure 6 Trade-off curves between $V_{\text {on }}$ and $E_{\text {off }}$ with different buffer doping

\section{CONCLUSION}

Simulation study was performed for SJ ITE-IGBT. The influence parameters on the trade-off performance of the device includes the position of local carrier lifetime control (LCLC) region, local carrier lifetime, and buffer layer doping level. For given local carrier lifetime, the position of LCLC region has an optimum range. Too near of LCLC region to buffer-collector junction may leads to high turnoff loss. The finite excess carrier recombination velocity in local carrier lifetime control region leads to the back-emitter injection efficiency $\gamma_{e}$ has a minimum value. This leads to the minimum $E_{\text {offmin }}$ and maximum $V_{\text {onmax }}$. And the longer local carrier lifetime is, the higher $E_{\text {offmin }}$ will be. Increasing 
buffer layer doping level appropriately could win better trade-off and wider trade-off range. All these results could provide helpful references for device design.

\section{ACKNOWLEDGMENT}

The authors wish to acknowledge Chinese National Natural Science Foundation Committee (Grant No. 61176071) and Ministry of Education Doctoral Discipline Specialized Research Fund (Grant No. 20111103120016) provides financial support.

\section{REFERENCES}

[1] B. J. Baliga, "Trends in power semiconductor devices", IEEE Trans. Electron Devices,vol. 43, pp1717-1731, Oct. 1996.

[2] X. B. Chen,"Semiconductor power device with alternationg conductivity type high-voltage breakdown regions", USA. Patent 5216275, 1993

[3] G. Deboy, M. Maerz, J.-P, Stenql, H. Strack J. Tihanyi, H. Weber, "New generation of high voltage MOSFEts breaks the limit line of silicon", International Elector Devices Meeting, pp683-685, 1998.

[4] X. B. Chen, P. A. Mawby, K. Board, C.A.T. Salama, "Theory of a novel voltage-sustaining layer for power devices", Microelectronics Journal, vol. 29, pp.1005-1011, Dec. 1998

[5] X.B. Chen, K. O. Sin, "Optimization of the specific on-resistance of the COOLMOS", IEEE Trans. Electron Devices, vol 48,pp344-348, Feb. 2001
[6] F. Bauer," The MOS contrlolled super junciton transistor(SJBR):a new highly efficient, high power semiconductor device for medium to high voltage applications", Proceedings of the International Symposium on Power Semiconductor Devices and ICs,pp.197-200, 2002

[7] K. H. Oh, J. Lee, Y. C. Kim, C. Yun, "A simulation sturdy on novel field stop IGBTs using superjunction”, IEEE Trans. Electron Devices, vol 53, pp884-890, Apr. 2006

[8] M. Antoniou, F. Udrea, F. Bauer, "Optimizaiton of super junction bipolar transistor for ultra-fast switching applications", Proceedings of the International Symposium on Power Semiconductor Devices and ICs, pp101-104, 2007

[9] M. Antoniou, F. Udrea, F. Bauer,'The superjunction insulated gate bipolar transistor optimization and modeling", IEEE Trans. Electron Devices, vol 57, pp.594-600, Mar. 2010

[10] F. Bauer, L. Nistor, A. Mihaila, M. Antoniou, F. Udrea, "Superjunciton IGBT filling the gap between SJ MOSFET and ultrafast IGBT", IEEE Electron Device Letters, vol 33, pp.1288-1290, Sep. 2012

[11] D. Hu, Y. Wu, B.W. Kang, X. You, X. Cheng, J.K.O Sin, “A new internal transparent collector IGBT", Proceedings of the International Symposium on Power Semiconductor Devices and ICs,pp287-298, 2009

[12] Taurus Workbench Manual, Avant! Corp. Fremont, CA, 1999

[13] F. Bauer, L. Nistor, A. Mihaila, F. Udrea, "Superjunction IGBTS:An evolutionary step of silicon power devices with high impact potential", Proceedings of the International Symposium on Power Semiconductor Devices and ICs, 00.27-36, 2012 